\title{
Vandermonde-form Preserving Matrices And The Generalized Signal Richness Preservation Problem
}

\author{
Borching $\mathrm{Su}$ \\ Department of Electrical Engineering \\ California Institute of Technology \\ Pasadena, California 91125 \\ Email: borching@ @ystems.caltech.edu
}

\author{
P. P. Vaidyanathan \\ Department of Electrical Engineering \\ California Institute of Technology \\ Pasadena, California 91125 \\ Email: ppvnath@systems.caltech.edu
}

\begin{abstract}
In this paper, a theoretical problem arising in digital communications, namely the generalized signal richness preservation problem, is addressed and studied. In order to solve the problem, a special class of square matrices, namely the "Vandermonde-form preserving" (VFP) matrices, is introduced and found to be highly relevant to the problem. Several properties of VFP matrices are studied in detail. The necessary and sufficient conditions of the problem have been found and a systematic proof is also presented.
\end{abstract}

\section{INTRODUCTION}

In digital communications, blind channel identification has been studied in the literature for a considerable period [11][14]. Many blind identification methods assume a special kind of redundancy in the input signal that facilitates blind identification. In particular, a method using linear redundant precoders with zero padding (ZP), proposed by Scaglione et al. [1], assumes the input signal to be rich. That is, for a sequence of $M \times 1$ vectors $\mathbf{s}(n), n \geq 0$, there exists a finite integer $J$ such that the $M \times J$ matrix

$$
\left[\begin{array}{llll}
\mathbf{s}(0) & \mathbf{s}(1) & \cdots & \mathbf{s}(J-1)
\end{array}\right]
$$

has full rank. Now, in some applications, the input signals are usually preconditioned by a linear transformation before being sent to the channel [9]. We are thus interested in whether the signal richness property is preserved after the linear transform. A theoretical treatment of the richness preservation problem has been presented in [10].

More recently, Manton et al. proposed another blind identification algorithm for transmitters using ZP that imposes less stringent conditions on input signals [2], [3], requiring only the coprimality property. A generalized algorithm has been proposed in [5] of which both blind identification methods mentioned above are special cases. The algorithm requires a generalized definition on signal richness with a parameter $Q$. When $Q=1$, it reduces to the conventional definition of richness. When $Q=M-1$, it becomes equivalent to the coprimality property stated in [3].

In this paper, we will focus on the theoretical issues of the generalized signal richness preservation problem and find out the necessary and sufficient conditions for linear precoders to preserve generalized signal richness. The rest of the paper is organized as follows. In Section II we give a definition of

\footnotetext{
${ }^{1}$ Work supported in parts by the NSF grant CCF-0428326 and the Moore Fellowship of the California Institute of Technology.
}

generalized signal richness and briefly describe several important properties thereof. The problem of preserving generalized signal richness will also be addressed. In Section III, the class of Vandermonde-form preserving (VFP) matrices will be introduced and several properties of VFP matrices will be studied in detail. In Section IV, the necessary and sufficient conditions for linear precoders to preserve generalized richness will be presented. Finally, Section V gives the conclusion and possible future directions. A journal version of this paper is under review [6].

\section{A. Notations}

Boldfaced lower case letters represent column vectors. Boldfaced upper case letters are reserved for matrices. Superscript $T$ as in $\mathbf{A}^{T}$ denotes the transpose operation of a matrix or a vector. $[\mathbf{v}]_{i}$ denotes the $i$ th element of vector $\mathbf{v}$, and $[\mathbf{A}]_{i j}$ denotes the entry at the $i$ th row and the $j$ th column of matrix A. All the vectors and matrices in this paper are complexvalued.

\section{Generalized Signal Richness And Problem FORMULATION}

\section{A. Definition of Generalized Signal Richness}

Definition 1: A sequence of $M \times 1$ vectors $\mathbf{s}(n), n \geq 0$, is said to be rich if there exists a finite integer $J$ such that the $M \times J$ matrix

$$
\left[\begin{array}{llll}
\mathbf{s}(0) & \mathbf{s}(1) & \cdots & \mathbf{s}(J-1)
\end{array}\right]
$$

has full row rank $M$.

The definition of the generalized signal richness for an $M \times 1$ signal will be given in Definition 3 as follows. We first build up the definition of a notation $\mathbf{s}_{Q}(n)$, representing a shifted and repeated version of $\mathbf{s}(n)$, using the following examples.

Example 1: $\mathbf{s}_{1}(n)$ is $\mathbf{s}(n)$ itself.

Example 2: Consider a sequence of $3 \times 1$ vectors $\mathbf{s}(n)$ defined as

$$
\left[\begin{array}{lll}
\mathbf{s}(0) & \mathbf{s}(1) & \mathbf{s}(2)
\end{array}\right]=\left[\begin{array}{lll}
1 & 4 & 7 \\
2 & 5 & 8 \\
3 & 6 & 9
\end{array}\right],
$$


and $\mathbf{s}(n)=0$ for $n \geq 3$. Then $\mathbf{s}_{2}(n)$ can be expressed as

$$
\begin{aligned}
& {\left[\begin{array}{llllll}
\mathbf{s}_{2}(0) & \mathbf{s}_{2}(1) & \mathbf{s}_{2}(2) & \mathbf{s}_{2}(3) & \mathbf{s}_{2}(4) & \mathbf{s}_{2}(5)
\end{array}\right] } \\
= & {\left[\begin{array}{llllll}
1 & 0 & 4 & 0 & 7 & 0 \\
2 & 1 & 5 & 4 & 8 & 7 \\
3 & 2 & 6 & 5 & 9 & 8 \\
0 & 3 & 0 & 6 & 0 & 9
\end{array}\right], }
\end{aligned}
$$

and $\mathbf{s}_{2}(n)=0$ for $n \geq 6$. And $\mathbf{s}_{3}(n)$ can be expressed as

$$
\begin{array}{r}
{\left[\mathbf{s}_{3}(0), \mathbf{s}_{3}(1), \mathbf{s}_{3}(2), \mathbf{s}_{3}(3), \mathbf{s}_{3}(4), \mathbf{s}_{3}(5), \mathbf{s}_{3}(6), \mathbf{s}_{3}(7), \mathbf{s}_{3}(8)\right]} \\
=\left[\begin{array}{llllllllll}
1 & 0 & 0 & 4 & 0 & 0 & 7 & 0 & 0 \\
2 & 1 & 0 & 5 & 4 & 0 & 8 & 7 & 0 \\
3 & 2 & 1 & 6 & 5 & 4 & 9 & 8 & 7 \\
0 & 3 & 2 & 0 & 6 & 5 & 0 & 9 & 8 \\
0 & 0 & 3 & 0 & 0 & 6 & 0 & 0 & 9
\end{array}\right],
\end{array}
$$

and $\mathbf{s}_{3}(n)=0$ for $n \geq 9$.

The formal definition of $\mathbf{s}_{Q}(n)$ is given as follows.

Definition 2: Given a positive integer $Q$ and a sequence of $M \times 1$ vectors $\mathbf{s}(n), \mathbf{s}_{Q}(n)$ is a sequence of $(M+Q-1) \times 1$ vectors defined as

$$
\mathbf{s}_{Q}(n Q+k)=\left[\begin{array}{c}
\mathbf{0}_{k \times 1} \\
\mathbf{s}(n) \\
\mathbf{0}_{(Q-k-1) \times 1}
\end{array}\right]
$$

for $n \geq 0, k=0,1, \ldots, Q-1$.

The definition of generalized signal richness is given as follows.

Definition 3: An $M \times 1$ sequence $\mathbf{s}(n), n \geq 0$ is said to be $(1 / Q)$-rich if $\mathbf{s}_{Q}(n)$ is rich.

Note that when $Q=1$, Definition 3 reduces to the conventional signal richness given in Definition 1. For the example given in Example 2, we can verify that $\mathbf{s}(n)$ is $(1 / 2)$ rich and (1/3)-rich but not 1 -rich.

An alternative definition of $(1 / Q)$-richness can be given immediately by using the following theorem.

Theorem $1((1 / Q)$-richness): Given an $M \times 1$ vector sequence $\mathbf{s}(n), n \geq 0, \mathbf{s}(n)$ is $(1 / Q)$-rich if and only if there does not exist a nonzero $Q \times M$ Hankel matrix $\mathbf{H}$ such that $\mathbf{H s}(n)=\mathbf{0}, \forall n \geq 0$.

Proof: See [6].

\section{B. Basic Properties of $(1 / Q)$-richness}

Several basic properties of $(1 / Q)$-richness are reviewed below, whose proofs have been presented in previous papers [5], [6].

Lemma 1: If a sequence of $M \times 1$ vectors $\mathbf{s}(n), n \geq 0$ is $(1 / Q)$-rich, then $\mathbf{s}(n)$ is $(1 /(Q+1))$-rich.

Lemma 2: If $M>1$ and an $M \times 1$ sequence $\mathbf{s}(n)$ is not $(1 /(M-1))$-rich, then it is not $(1 / Q)$-rich for any $Q$.

Proof: See [5].

Lemma 1 states a basic property of generalized signal richness: the smaller the value of $Q$ is, the "stronger" the condition of $(1 / Q)$-richness is. We can thus define a measure of generalized signal richness, namely the degree of non-richness for a given $M \times 1$ sequence $\mathbf{s}(n)$ as follows.

Definition 4: Given an $M \times 1$ sequence $\mathbf{s}(n), n \geq 0$, the degree of non-richness of $\mathbf{s}(n)$ is defined as:

$$
Q_{\min } \triangleq \min _{Q}\left(\mathbf{s}(n) \text { is } \frac{1}{Q} \text {-rich }\right) \text {. }
$$

If $\mathbf{s}(n)$ is not $(1 / Q)$-rich for any $Q$, then $Q_{\text {min }}=\infty$. With Lemma 2, we can see that for an $M \times 1$ sequence $\mathbf{s}(n),(1 /(M-1))$-richness is the weakest form of generalized richness. Given a $M \times 1$ vector sequence $\mathbf{s}(n)$, the degree of non-richness can only be one of values $1,2, \ldots, M-1$, or $\infty$.

An $M \times 1$ sequence $\mathbf{s}(n)$ has an infinite degree of nonrichness if and only if there exists an $(M+Q-1)$-element row vector $\mathbf{v}^{T}$ such that $\mathbf{v}^{T} \mathbf{s}_{Q}(n)=0$ for any $Q$. This is true in particular when an $M$-element row vector in the form

$$
\mathbf{w}^{T}=c\left[\begin{array}{lllll}
1 & \alpha & \alpha^{2} & \cdots & \alpha^{M-1}
\end{array}\right]
$$

is an annihilator of $\mathbf{s}(n)$ (i.e., $\mathbf{w}^{T} \mathbf{s}(n)=0$ ). In this case we can show that

$$
\mathbf{v}^{T}=\left[\begin{array}{lllll}
1 & \alpha & \alpha^{2} & \cdots & \alpha^{M+Q-2}
\end{array}\right]
$$

is an annihilator of $\mathbf{s}_{Q}(n)$. In another situation, if an $M$ element row vector

$$
\mathbf{w}^{T}=\left[\begin{array}{llll}
0 & \cdots & 0 & c
\end{array}\right]
$$

is an annihilator of $\mathbf{s}(n)$, it can also be readily shown that $\mathbf{s}(n)$ has an infinite degree of non-richness. Furthermore, it is shown [5] that if $\mathbf{s}(n)$ has an infinite degree of non-richness, there must exist an $M$-element row vector in a form of either Eq. (2) or Eq. (3) so that it is an annihilator of $\mathbf{s}(n)$. We can give a unifying definition to row vectors in forms of Eqs. (2) and (3) as follows.

Definition 5 (Vandermonde Form Vectors): A row vector $\mathbf{v}^{T}=\left[\begin{array}{llll}v_{1} & v_{2} & \cdots & v_{M}\end{array}\right]$ is said to be in the "Vandermonde form" if there exist $\alpha, \beta \in \mathbb{C},|\alpha|^{2}+|\beta|^{2}>0$, such that

$$
\mathbf{v}^{T}=\left[\begin{array}{lllll}
\beta^{M-1} & \alpha \beta^{M-2} & \cdots & \alpha^{M-2} \beta & \alpha^{M-1}
\end{array}\right] .
$$

The "Vandermonde ratio" $\gamma \in \mathbb{C} \bigcup\{\infty\}$ of $\mathbf{v}^{T}$ is defined as

$$
\gamma=\left\{\begin{array}{ll}
\frac{\alpha}{\beta} & \text { if } \beta \neq 0 \\
\infty & \text { if } \beta=0
\end{array} .\right.
$$

The set of all $M$-vectors in Vandermonde form, denoted as $\mathcal{V}_{M}$, is defined as

$\mathcal{V}_{M}=\left\{\mathbf{v}^{T} \mid \mathbf{v} \in \mathbb{C}^{M}\right.$ and $\mathbf{v}^{T}$ is in the Vandermonde form $\}$

Using this definition, the properties of $(1 / Q)$-richness discussed above can be summarized in the following lemma.

Lemma 3: Consider a sequence of $M \times 1$ vector $\mathbf{s}(n), n \geq$ 0 . The following statements are equivalent:

1) $\mathbf{s}(n)$ is not $(1 / Q)$-rich for any $Q$.

2) The degree of non-richness of $\mathbf{s}(n)$ is infinity. 
3) There exists a Vandermonde form vector $\mathbf{v}^{T} \in \mathcal{V}_{M}$ (with a Vandermonde ratio $\gamma \in \mathbb{C} \bigcup\{\infty\})$ such that $\mathbf{v}^{T} \mathbf{s}(n)=$ $0, \forall n \geq 0$.

\section{Main Problem}

Definition 6: An $M \times M$ matrix $\mathbf{R}$ is said to be $(1 / Q)$ richness preserving if and only if for any $(1 / Q)$-rich signal $\mathbf{s}(n)$, the output $\mathbf{u}(n)=\mathbf{R} \mathbf{s}(n)$ is also a $(1 / Q)$-rich signal.

The problem of interest in this paper is to find the necessary and sufficient conditions for $M \times M$ matrices that preserves $(1 / Q)$-richness. Since $1 /(M-1)$-richness is the weakest form of generalized signal richness for an $M \times 1$ vector sequence (as depicted in Lemma 2), we will solve this problem for cases when $1 \leq Q \leq M-1$. For the case when $Q=1$, it is obvious that an $M \times M$ constant matrix $\mathbf{R}$ preserves 1-richness if and only if $\mathbf{R}$ is nonsingular. However, in the case when $Q>1$, a nonsingular matrix $\mathbf{R}$ does not necessarily preserve $(1 / Q)$-richness. This can be seen in the following simple example.

Example 3: Let $\mathbf{s}(0)=\left[\begin{array}{lll}1 & -1 & 0\end{array}\right]^{T}, \mathbf{s}(1)=$ $\left[\begin{array}{lll}1 & 1 & 2\end{array}\right]^{T}$, and $\mathbf{s}(n)=\mathbf{0}$ for $n>1$. By observing that

$$
\mathbf{A}=\left[\begin{array}{cccc}
1 & 0 & 1 & 0 \\
-1 & 1 & 1 & 1 \\
0 & -1 & 2 & 1 \\
0 & 0 & 0 & 2
\end{array}\right]
$$

has full rank 4, we know that $\mathbf{s}(n)$ is (1/2)-rich. Now let

$$
\mathbf{R}=\left[\begin{array}{lll}
1 & 0 & 0 \\
0 & 0 & 1 \\
0 & 1 & 0
\end{array}\right]
$$

which is an invertible permutation matrix. Then we can obtain the output $\mathbf{u}(n)=\mathbf{R s}(n)$ as $\mathbf{u}(0)=\left[\begin{array}{lll}1 & 0 & -1\end{array}\right]^{T}$ and $\mathbf{u}(1)=\left[\begin{array}{lll}1 & 2 & 1\end{array}\right]^{T}$. Note that if $\mathbf{v}^{T}=\left[\begin{array}{lll}1 & -1 & 1\end{array}\right]$, then $\mathbf{v}^{T} \mathbf{u}(n)=0$ for all $n$. So $\mathbf{u}(n)$ is not $(1 / Q)$-rich for any $Q$. This suggests that an invertible constant precoder, although preserving the "rank" of a signal, does not preserve $(1 / Q)$ richness in general!

\section{VANDERMONDE-FORM PRESERVING MATRICES}

In this section we introduce a new class of square matrices, namely the Vandermonde-form preserving (VFP) matrices. We will study several important properties of VFP matrices which are useful for solving the main problem described in the previous section.

\section{A. Preservation of $1 /(M-1)$-richness}

Definition 7: An $M \times M$ constant matrix $\mathbf{R}$ is said to be a "Vandermonde-form preserving" (VFP) matrix if $\mathbf{v}^{T} \mathbf{R} \in \mathcal{V}_{M}$ for all $\mathbf{v}^{T} \in \mathcal{V}_{M}$.

It is not difficult to verify that $M \times M$ VFP matrices are $1 /(M-1)$-richness preserving. Consider an $M \times M$ VFP matrix $\mathbf{R}$ and an $M \times 1$ vector sequence $\mathbf{s}(n)$ which is $1 /(M-$ 1)-rich. Assume $\mathbf{R}$ is not $1 /(M-1)$-richness preserving and hence $\mathbf{u}(n)=\mathbf{R s}(n)$ is not $1 /(M-1)$-rich. Then by Lemma
3 there exists $\mathbf{v}^{T} \in \mathcal{V}_{M}$ such that $\mathbf{v}^{T} \mathbf{u}(n)=0$. This implies $\mathbf{v}^{T} \mathbf{R s}(n)=0$. Since $\mathbf{R}$ is a VFP matrix, $\mathbf{v}^{T} \mathbf{R}$ is also in Vandermonde form. This contradicts the assumption that $\mathbf{s}(n)$ is $1 /(M-1)$-rich.

On the contrary, being a VFP matrix is also a necessary condition for $\mathbf{R}$ to preserve $1 /(M-1)$-richness. In fact, if $\mathbf{R}$ is not a VFP matrix, we can always construct a $(1 / 2)$-rich signal $\mathbf{s}(n)$ such that $\mathbf{u}(n)=\mathbf{R} \mathbf{s}(n)$ has an infinite degree of non-richness, as shown in the following lemma.

Lemma 4: For $M>1$, consider an $M \times M$ matrix $\mathbf{R}$. If $2 \leq Q \leq M-1$ and $\mathbf{R}$ is $(1 / Q)$-richness preserving, then $\mathbf{R}$ must be VFP.

Proof: See Appendix.

Summarizing these arguments, we obtain the following theorem, which solves the main problem for the case when $Q=M-1$.

Theorem 2: An $M \times M$ matrix $\mathbf{R}$ preserves $1 /(M-1)$ richness if and only if $\mathbf{R}$ is VFP.

\section{B. Representation of Vandermonde-form preserving Matrices}

Before we proceed to solve the main problem for other $Q$ 's, we want to first find out what VFP matrices look like. Obviously the identity matrix $\mathbf{I}_{M}$ and any nonzero multiple of it are VFP matrices. A permutation matrix, however, is in general not a VFP matrix, such as the one given in Example 3. So is there any VFP matrix other than a multiple of an identity matrix? The following theorem gives the most general representation of VFP matrices.

Theorem 3: An $M \times M$ matrix $\mathbf{R}=\left[\begin{array}{llll}\mathbf{r}_{1} & \mathbf{r}_{2} & \cdots & \mathbf{r}_{M}\end{array}\right]$ is Vandermonde-form preserving if and only if there exists a $2 \times 2$ invertible matrix

$$
\mathbf{R}_{2}=\left[\begin{array}{ll}
a & b \\
c & d
\end{array}\right]
$$

such that

$$
r_{k}(x)=(a+c x)^{M-k}(b+d x)^{k-1}, k=1,2, \ldots, M
$$

where $r_{k}(x)$ is the polynomial representation of the column vector $\mathbf{r}_{k}$, i.e., $r_{k}(x)=\left[\begin{array}{llll}1 & x & \cdots & x^{M-1}\end{array}\right] \mathbf{r}_{k}$. The $2 \times 2$ matrix $\mathbf{R}_{2}$ is called the characteristic matrix of the $M \times M$ VFP matrix $\mathbf{R}$.

Proof: See [6].

Theorem 3 essentially gives us a construction method for an $M \times M$ VFP matrix using a "seed" $2 \times 2$ nonsingular matrix $\mathbf{R}_{2}$ defined in Eq. (4). Note that $\mathbf{R}_{2}$ is always a VFP matrix as long as it is nonsingular (i.e., $a d-b c \neq 0$ ) since a $1 \times 2$ nonzero vector is always in the Vandermonde form. Besides, we can see that any $M \times M$ VFP matrix $\mathbf{R}_{M}$ can be parameterized by a $2 \times 2$ Vandermonde-form preserving matrix. Thus the number of freedoms of $M \times M$ Vandermonde-form preserving matrices is always a constant for any $M>1$. For convenience, we denote

$$
\mathcal{R}_{M}\left(\left[\begin{array}{ll}
a & b \\
c & d
\end{array}\right]\right)
$$

where $a d-b c \neq 0$, as the $M \times M$ Vandermonde-form preserving matrix generated with polynomials $a+c x$ and 
$b+d x$. For example,

$$
\mathcal{R}_{3}\left(\left[\begin{array}{ll}
a & b \\
c & d
\end{array}\right]\right)=\left[\begin{array}{ccc}
a^{2} & a b & b^{2} \\
2 a c & a d+b c & 2 b d \\
c^{2} & c d & d^{2}
\end{array}\right] .
$$

Some more numerical examples are presented below for a better understanding of VFP matrices.

$$
\begin{aligned}
& \text { Example 4: If we choose } \mathbf{R}_{2}=\left[\begin{array}{ll}
1 & 1 \\
0 & 1
\end{array}\right] \text {, then } \\
& \mathbf{R}_{3}=\left[\begin{array}{lll}
1 & 1 & 1 \\
0 & 1 & 2 \\
0 & 0 & 1
\end{array}\right] \text { and } \mathbf{R}_{4}=\left[\begin{array}{llll}
1 & 1 & 1 & 1 \\
0 & 1 & 2 & 3 \\
0 & 0 & 1 & 3 \\
0 & 0 & 0 & 1
\end{array}\right] .
\end{aligned}
$$

A VFP matrix can also be a full matrix. If we choose $\mathbf{R}_{2}=$

$$
\begin{aligned}
& {\left[\begin{array}{ll}
1 & 2 \\
1 & 1
\end{array}\right] \text {, then }} \\
& \mathbf{R}_{3}=\left[\begin{array}{lll}
1 & 2 & 4 \\
2 & 3 & 4 \\
1 & 1 & 1
\end{array}\right] \text { and } \mathbf{R}_{4}=\left[\begin{array}{cccc}
1 & 2 & 4 & 8 \\
3 & 5 & 8 & 12 \\
3 & 4 & 5 & 6 \\
1 & 1 & 1 & 1
\end{array}\right] .
\end{aligned}
$$

\section{Vandermonde Ratio Transformation}

Theorem 4: Suppose $\mathbf{v}^{T} \in \mathcal{V}_{M}$ has a Vandermonde ratio $\alpha \in \mathbb{C} \bigcup\{\infty\}$ and $\mathbf{R}_{M}$ is a VFP matrix with a nonsingular characteristic matrix

$$
\mathbf{R}_{2}=\left[\begin{array}{ll}
a & b \\
c & d
\end{array}\right]
$$

Then $\mathbf{w}^{T}=\mathbf{v}^{T} \mathbf{R}_{M}$ is also a Vandermonde form vector with Vandermonde ratio $\beta=f(\alpha)$ where $f: \mathbb{C} \bigcup\{\infty\} \rightarrow \mathbb{C} \bigcup\{\infty\}$ is called the characteristic function of $\mathbf{R}_{M}$, defined as

$$
f(\alpha)=\lim _{x \rightarrow \alpha} \frac{b+d x}{a+c x} .
$$

Proof: See [6].

Some numerical examples are presented below to demonstrate Theorem 4 and clarify the concept.

Example 5: We take $\mathbf{R}_{2}=\left[\begin{array}{ll}1 & 2 \\ 1 & 1\end{array}\right]$ as in Example 4.

Then the $4 \times 4$ VFP matrix characterized by $\mathbf{R}_{2}$ is

$$
\mathbf{R}_{4}=\left[\begin{array}{cccc}
1 & 2 & 4 & 8 \\
3 & 5 & 8 & 12 \\
3 & 4 & 5 & 6 \\
1 & 1 & 1 & 1
\end{array}\right]
$$

The characteristic function of $\mathbf{R}_{4}$ is

$$
f(\alpha)=\lim _{x \rightarrow \alpha} \frac{2+x}{1+x} .
$$

Let $\mathbf{v}^{T}=\left[\begin{array}{llll}1 & -3 & 9 & -27\end{array}\right]$, which has a Vandermonde ratio $\alpha=-3$. Then

$$
\mathbf{w}^{T}=\mathbf{v}^{T} \mathbf{R}_{4}=\left[\begin{array}{llll}
-8 & -4 & -2 & -1
\end{array}\right]
$$

has a Vandermonde ratio $\beta=f(\alpha)=(2-3) /(1-3)=1 / 2$.
If $\mathbf{v}^{T}=\left[\begin{array}{llll}1 & -1 & 1 & -1\end{array}\right]$, which has a Vandermonde ratio $\alpha=-1$, then

$$
\mathbf{w}^{T}=\mathbf{v}^{T} \mathbf{R}_{4}=\left[\begin{array}{llll}
0 & 0 & 0 & 1
\end{array}\right]
$$

has a Vandermonde ratio $\beta=f(\alpha)=\infty$.

If $\mathbf{v}^{T}=\left[\begin{array}{llll}0 & 0 & 0 & 1\end{array}\right]$, which has a Vandermonde ratio $\alpha=\infty$, then

$$
\mathbf{w}^{T}=\mathbf{v}^{T} \mathbf{R}_{4}=\left[\begin{array}{llll}
1 & 1 & 1 & 1
\end{array}\right]
$$

has a Vandermonde ratio $\beta=f(\alpha)=1 / 1=1$.

From the discussions above, we find that a VFP matrix "bilinearly" transforms the Vandermonde ratio of a Vandermonde form vector with the characteristic function $f$ defined in Theorem 4. Note that the function $f$ is a one-to-one and onto function. The inverse function of $f$ can be expressed as

$$
g(\beta)=\lim _{y \rightarrow \beta}\left(-\frac{a y-b}{c y-d}\right) .
$$

\section{Hankel-form Preservation}

Another interesting property of VFP matrices is the following.

Theorem 5 (Hankel-form Preservation): Given an $m \times n$ nonzero Hankel matrix $\mathbf{H}=\left[h_{i j}\right]$. Let $\mathbf{R}_{2}$ be a $2 \times 2$ invertible matrix. Let $\mathbf{R}_{m}=\mathcal{R}_{m}\left(\mathbf{R}_{2}\right)$ and $\mathbf{R}_{n}=\mathcal{R}_{n}\left(\mathbf{R}_{2}\right)$ be $m \times m$ and $n \times n$ VFP matrices, respectively (the notation $\mathcal{R}_{M}(\cdot)$ was defined in Section III-B). Then $\mathbf{H}^{\prime}=\mathbf{R}_{m}^{T} \mathbf{H} \mathbf{R}_{n}$ is also a nonzero Hankel matrix.

Proof: See Appendix.

Theorem 5 shows another capability of VFP matrices: besides preserving Vandermonde form vectors, they also preserve the property of Hankel matrices if we use two VFP matrices with the same characteristic matrix. An example is shown below.

$$
\begin{aligned}
& \text { Example 6: Let } \mathbf{R}_{2}=\left[\begin{array}{ll}
1 & 1 \\
2 & 0
\end{array}\right], \mathbf{R}_{3}=\mathcal{R}_{3}\left(\mathbf{R}_{2}\right) \text {, and } \\
& \mathbf{H}=\left[\begin{array}{lll}
h_{1} & h_{2} & h_{3} \\
h_{2} & h_{3} & h_{4}
\end{array}\right]
\end{aligned}
$$

be a nonzero Hankel matrix. Then

$$
\begin{aligned}
& \mathbf{H}^{\prime}=\mathbf{R}_{2}^{T} \mathbf{H} \mathbf{R}_{3} \\
= & {\left[\begin{array}{ll}
1 & 2 \\
1 & 0
\end{array}\right]\left[\begin{array}{lll}
h_{1} & h_{2} & h_{3} \\
h_{2} & h_{3} & h_{4}
\end{array}\right]\left[\begin{array}{ccc}
1 & 1 & 1 \\
4 & 2 & 0 \\
4 & 0 & 0
\end{array}\right] } \\
= & {\left[\begin{array}{ccc}
h_{1}+6 h_{2}+12 h_{3}+8 h_{4} & h_{1}+4 h_{2}+4 h_{3} & h_{1}+2 h_{2} \\
h_{1}+4 h_{2}+4 h_{3} & h_{1}+2 h_{2} & h_{1}
\end{array}\right] }
\end{aligned}
$$

is also a nonzero Hankel matrix.

\section{MAIN THEOREM}

Now we are ready to solve the problem stated in Section II-C. Using Theorem 5 and Lemma 4, the problem can now be completely answered by the following theorem.

Theorem 6: (1/Q-richness Preservation) For $M>1,2 \leq$ $Q \leq M-1$, an $M \times M$ matrix $\mathbf{R}_{M}$ is $(1 / Q)$-richness preserving if and only if $\mathbf{R}_{M}$ is Vandermonde-form preserving.

Proof: The necessity comes directly from Lemma 4. As for sufficiency, suppose a Vandermonde-form preserving 
matrix $\mathbf{R}_{M}=\mathcal{R}_{M}\left(\mathbf{R}_{2}\right)$ is not $(1 / Q)$-richness preserving for some $Q \geq 2$, where $\mathbf{R}_{2}$ is a $2 \times 2$ invertible matrix. Then there exists a $(1 / Q)$-rich signal $\mathbf{s}(n)$ such that the output $\mathbf{u}(n)=\mathbf{R}_{M} \mathbf{s}(n)$ is not $(1 / Q)$-rich. Using Theorem 1, there exists a $Q \times M$ nonzero Hankel matrix $\mathbf{H}$ such that $\mathbf{H u}(n)=\mathbf{0}$ for all $n \geq 0$. This implies $\mathbf{H R}_{M} \mathbf{s}(n)=\mathbf{0}$ for all $n \geq 0$. Let $\mathbf{R}_{Q}=\mathcal{R}_{Q}\left(\mathbf{R}_{2}\right)$. We have $\mathbf{R}_{Q}^{T} \mathbf{H} \mathbf{R}_{M} \mathbf{s}(n)=\mathbf{0}$ for all $n \geq 0$. Using Theorem 5, we know that $\mathbf{R}_{Q}^{T} \mathbf{H} \mathbf{R}_{M}$ is also a Hankel matrix. Now using Theorem 1 again, we conclude that $\mathbf{s}(n)$ is also not $(1 / Q)$-rich, contradicting the assumption that it is $(1 / Q)$-rich. So a Vandermonde-form preserving matrix must be $(1 / Q)$-richness preserving for $Q \geq 2$.

A summary of the answer of the main problem is given as follows. Given an $M \times M$ matrix $\mathbf{R}$, then

1) when $Q=1, \mathbf{R}$ preserves $(1 / Q)$-richness if and only if $\mathbf{R}$ is nonsingular;

2) when $2 \leq Q \leq M-1, \mathbf{R}$ preserves $(1 / Q)$-richness if and only if $\mathbf{R}$ is a VFP matrix.

\section{Concluding Remarks}

In this paper, we described a mathematical problem that arises in some applications on blind channel identification. We introduced Vandermonde-form preserving (VFP) matrices as a new subclass of invertible matrices which are highly relevant to the problem. Several properties of VFP matrices have been presented clearly and the proof of the answer to the problem has been presented systematically.

In the future, it may be useful to consider the problem in general for a system with memory, in which case the transfer function of the precoder is an $M \times M$ polynomial matrix $\mathbf{R}(z)=\sum_{k=0}^{N} \mathbf{r}(k) z^{-k}$. It is also of interest to deal with a rectangular $P \times M$ system $\mathbf{R}(z)$. Finding other engineering applications of VFP matrices will also be interesting.

\section{APPENDIX}

Proof of Lemma 4: Assume $\mathbf{R}$ is not VFP. Then there exists $\mathbf{v}^{T} \in \mathcal{V}_{M}$ such that $\mathbf{w}^{T}=\mathbf{v}^{T} \mathbf{R} \notin \mathcal{V}_{M}$. Construct a vector sequence $\mathbf{s}(n), n \geq 0$ as follows. Let $\mathbf{s}(0), \mathbf{s}(1), \cdots, \mathbf{s}(M-2)$ be selected as $(M-1)$ linearly independent column vectors that are orthogonal to $\mathbf{w}^{T} \notin \mathcal{V}_{M}$. Let $\mathbf{s}(n)=\mathbf{0}$ for all $n \geq$ $M-1$. Since $\mathbf{w}^{T} \notin \mathcal{V}_{M}$ is the only annihilator of $\mathbf{s}(n)$, there does not exist a $2 \times M$ nonzero Hankel matrix $\mathbf{H}$ such that $\mathbf{H s}(n)=\mathbf{0}$. So $\mathbf{s}(n)$ is $(1 / 2)$-rich and hence is $(1 / Q)$-rich for any $Q \geq 2$. Now consider $\mathbf{u}(n)=\boldsymbol{R} \mathbf{s}(n)$. We have $\mathbf{v}^{T} \mathbf{u}(n)=$ $\mathbf{v}^{T} \mathbf{R} \mathbf{s}(n)=\mathbf{w}^{T} \mathbf{s}(n)=\mathbf{0}$. By Lemma $3, \mathbf{u}(n)$ is not $1 / Q$ rich for any $Q$. So $\mathbf{R}$ is not $(1 / Q)$-richness preserving for any $Q \geq 2$.

The proof of Theorem 5 requires the following lemma.

Lemma 5: Let $\mathbf{H}$ be an $m \times n$ Hankel matrix whose entry values come from an $(m+n-1) \times 1$ vector $\mathbf{h}$. That is, $[\mathbf{H}]_{i j}=[\mathbf{h}]_{i+j-1}=h_{i+j-1}$. Let $\mathbf{u}$ and $\mathbf{v}$ be $m \times 1$ and $n \times 1$ column vectors, respectively. Then $\mathbf{u}^{T} \mathbf{H v}=\mathbf{w}^{T} \mathbf{h}$, where $\mathbf{w}$ is an $(m+n-1)$-vector whose entries come from convolution of $\mathbf{u}$ and $\mathbf{v}$ :

$$
[\mathbf{w}]_{k}=\sum_{l=1}^{m}[\mathbf{u}]_{l}[\mathbf{v}]_{k-l+1} .
$$

Proof: The Lemma is immediately verified by observing that the coefficient associated with $h_{k}$ in the sum $\mathbf{u}^{T} \mathbf{H v}$ is $\sum_{l=1}^{m} u_{l} v_{k-l+1}$. (Assuming $v_{l}=0$ when $l \leq 0$ or $l>n$.)

Proof of Theorem 5: Denote the $k$ th column of $\mathbf{R}_{m}$ as $\mathbf{r}_{m, k}$ and the $l$ th column of $\mathbf{R}_{n}$ as $\mathbf{r}_{n, l}$. Let $r_{m k}(x)=\left[\begin{array}{cccc}1 & x & \cdots & x^{m-1}\end{array}\right] \mathbf{r}_{m, k}$ and $r_{n l}(x)=$ $\left[\begin{array}{llll}1 & x & \cdots & x^{n-1}\end{array}\right] \mathbf{r}_{n, l}$. From construction of VFP matrices we know $r_{m k}(x)=(a+c x)^{m-k}(b+d x)^{k-1}$ and $r_{n l}(x)=(a+c x)^{n-l}(b+d x)^{l-1}$. The $k l$-th entry of $\mathbf{H}^{\prime}$, $\left[\mathbf{H}^{\prime}\right]_{k l}$, can be expressed as $\mathbf{r}_{m, k}^{T} \mathbf{H r}_{n, l}$. Using Lemma 5, we have

$$
\left[\mathbf{H}^{\prime}\right]_{k l}=\mathbf{w}_{k, l}^{T} \mathbf{h},
$$

where the polynomial representation of the $(m+n-1) \times 1$ vector $\mathbf{w}_{k, l}$ is $w_{k l}(x)=r_{m k}(x) r_{n l}(x)=(a+c x)^{m+n-k-l}(b+$ $d x)^{k+l-2}$. The polynomial $w_{k l}(x)$ stays unchanged when $k+l$ is fixed. So from Eq. (7), the value of $\left[\mathbf{H}^{\prime}\right]_{k l}$ is a function of $(k+l)$ and hence $\mathbf{H}^{\prime}$ is also a Hankel matrix. $\mathbf{H}^{\prime}$ being nonzero is readily verified by observing that both $\mathbf{R}_{n}$ and $\mathbf{R}_{m}$ are invertible.

\section{REFERENCES}

[1] A. Scaglione, G. B. Giannakis, and S. Barbarossa, "Redundant filter bank precoders and equalizers Part II: Synchronization and direct equalization”, IEEE Trans. SP, vol. 47, pp. 2007-2002, Jul. 1999.

[2] J. H. Manton, W. D. Neumann, "Totally Blind Channel Identification by Exploiting Guard Intervals", Systems and Control Letters, vol. 48, no. 2, pp. 113-119, 2003.

[3] D. H. Pham and J. H. Manton, "A Subspace Algorithm for Guard Interval Based Channel Identification and Source Recovery Requiring Just Two Received Blocks", Proc. of ICASSP 2003, pp. 317 - 320, Hong Kong, China.

[4] B. Su and P. P. Vaidyanathan, "A Generalization of Deterministic Algorithm for Blind Channel Identification with Filter Bank Precoders", Proc. of ISCAS 2006, Kos Island, Greece.

[5] B. Su and P. P. Vaidyanathan, "A Generalized Algorithm for Blind Channel Identification with Linearly Redundant Precoding," Submitted to EURASIP Journal on Applied Signal Processing Special Issue on Multirate Systems.

[6] B. Su and P. P. Vaidyanathan, "Generalized Signal Richness Preservation Problem and Vandermonde-form Preserving Matrices," Submitted to IEEE Transactions on Signal Procssing.

[7] D. E. Knuth, The Art of Computer Programming Vol. 2, 3rd Edition, Addison Wesley, 1997.

[8] G. H. Golub and C. F. Van Loan, Matrix Computations, 3rd Edition, Johns Hopkins University Press, 1996.

[9] S.-M. Phoong and K.-Y. Chang, "Antipodal paraunitary matrices and their application to OFDM systems", IEEE Trans. Signal Processing, vol. 53, pp. 1374-1386, April 2005.

[10] B. Su and P. P. Vaidyanathan, "Theoretical Issues on LTI Systems Which Preserver Signal Richness" IEEE Trans. Signal Processing, in press.

[11] B. Porat and B. Friedlander, "Blind equalization of digital communication channels using high-order moments," IEEE Trans. SP, vol. 39, no. 2, pp. 522-526, Feb. 1991.

[12] L. Tong, G. Xu and T. Kailath, "A new approach to blind identification and equalization of multipath channels," Proc. 25th Asilomar Conf. on Signals, Systems, and Computers, Nov. 1991.

[13] Y. Li and Z. Ding, "Blind channel identification based on second order cyclostationary statistics," Proc. of ICASSP 1993, pp. 81-84, Minneapolis, MN.

[14] E. Moulines, P. Duhamel, J. F. Cardoso, S. Mayrargue, "Subspace Methods for the Blind Identification of Multichannel FIR Filters," IEEE Trans. on SP, pp. 516-525, Feb. 1995. 